\title{
Detection of clinical mastitis with sensor data from automatic milking systems is improved by using decision-tree induction
}

\author{
C. Kamphuis, ${ }^{* 1}$ H. Mollenhorst, ${ }^{*}$ J. A. P. Heesterbeek, ${ }^{*}$ and H. Hogeveen ${ }^{*} \dagger$ \\ *Department of Farm Animal Health, Faculty of Veterinary Medicine, Utrecht University, Utrecht, the Netherlands \\ †Chair Group of Business Economics, Wageningen University, Wageningen, the Netherlands
}

\begin{abstract}
The objective was to develop and validate a clinical mastitis (CM) detection model by means of decisiontree induction. For farmers milking with an automatic milking system (AMS), it is desirable that the detection model has a high level of sensitivity (Se), especially for more severe cases of CM, at a very high specificity ( $\mathrm{Sp}$ ). In addition, an alert for CM should be generated preferably at the quarter milking (QM) at which the CM infection is visible for the first time. Data were collected from 9 Dutch dairy herds milking automatically during a 2.5-yr period. Data included sensor data (electrical conductivity, color, and yield) at the QM level and visual observations of quarters with CM recorded by the farmers. Visual observations of quarters with CM were combined with sensor data of the most recent automatic milking recorded for that same quarter, within a $24-\mathrm{h}$ time window before the visual assessment time. Sensor data of 3.5 million QM were collected, of which 348 QM were combined with a CM observation. Data were divided into a training set, including two-thirds of all data, and a test set. Cows in the training set were not included in the test set and vice versa. A decision-tree model was trained using only clear examples of healthy $(\mathrm{n}=24,717)$ or diseased $(\mathrm{n}=243)$ QM. The model was tested on $105 \mathrm{QM}$ with $\mathrm{CM}$ and a random sample of 50,000 QM without CM. While keeping the Se at a level comparable to that of models currently used by AMS, the decision-tree model was able to decrease the number of false-positive alerts by more than $50 \%$. At an Sp of $99 \%, 40 \%$ of the CM cases were detected. Sixty-four percent of the severe CM cases were detected and only $12.5 \%$ of the CM that were scored as watery milk. The Se increased considerably from $40 \%$ to $66.7 \%$ when the time window increased from less than $24 \mathrm{~h}$ before the CM observation, to a time window from 24 $\mathrm{h}$ before to $24 \mathrm{~h}$ after the CM observation. Even at very wide time windows, however, it was impossible to reach
\end{abstract}

Received March 4, 2010.

Accepted April 13, 2010.

${ }^{1}$ Corresponding author: C.Kamphuis@uu.nl an Se of $100 \%$. This indicates the inability to detect all CM cases based on sensor data alone. Sensitivity levels varied largely when the decision tree was validated per herd. This trend was confirmed when decision trees were trained using data from 8 herds and tested on data from the ninth herd. This indicates that when using the decision tree as a generic CM detection model in practice, some herds will continue having difficulties in detecting CM using mastitis alert lists, whereas others will perform well.

Key words: clinical mastitis detection, robotic milking, sensor data, decision-tree induction

\section{INTRODUCTION}

Mastitis is one of the most frequent and costly diseases in dairy cows (e.g., Halasa et al., 2007). Detecting clinical mastitis (CM) is important to maintain an acceptable level of milk quality, to initiate an antibiotic treatment when necessary, and to safeguard the welfare of a dairy herd (Kamphuis et al., 2008b). When using automatic milking systems (AMS), farmers are not present during the milking process to check the cow and the milk visually for CM. Instead, they detect cases of CM by means of several information sources. The mastitis alert list, noting those cows and quarters that are likely to have $\mathrm{CM}$, is one of these information sources. These lists are the output of a CM detection model available at the AMS. As input for such a detection model, the AMS uses in-line sensor information, with electrical conductivity being the trait most commonly measured in-line. Although farmers who use AMS are able to manage udder health sufficiently (de Koning et al., 2004), udder health is under pressure on farms with AMS (Dohmen et al., 2010). One way to improve udder health on farms with an AMS is to improve the sensitivity (Se) of the currently available mastitis detection models. In addition, specificity (Sp) levels need improvement, as farmers are eager to minimize the additional labor of checking large numbers of falsepositive alerts, even at the expense of missing some true cases of CM (Claycomb et al., 2009). A level of $99 \%$ for the Sp and a minimum level of $70 \%$ for the Se 
have been suggested as goal for CM detection models when applied in practice (Mein and Rasmussen, 2008).

Several models to detect mastitis using sensor information have been developed in the past and some studies have reported high levels of Se and Sp. However, few of these models are implemented in a practical AMS setting. There are several reasons why these models may not be applied in practice, such as the wide time windows used in some studies and the fact that some models were trained and validated using only clear cases of healthy and mastitic quarters or cows. Some of the earlier studies developing a mastitis detection model based on electrical conductivity information used very long time windows; for example, de Mol et al. (1997) used a time window of $10 \mathrm{~d}$ before the actual CM case until $7 \mathrm{~d}$ after. Using wide time windows will result in models showing a good detection performance (Sherlock et al., 2008), but farmers need a CM alert within a very limited period before or only at the milking when CM occurs (Hogeveen and Ouweltjes, 2003). Friggens et al. (2007) developed and validated their CM detection models with highly selected data including only those cows that clearly had CM and those that were clearly healthy. Such validation, however, will overestimate the detection performance of the model because field data include data from cows or (quarter) milkings that have a less clear mastitis status as well. A CM detection model should be able to deal with this so-called gray area.

One of the major problems of developing a CM detection model using sensor data is that the data are often noisy and incomplete. In addition, the low prevalence of CM results in highly imbalanced data, which makes modeling even more difficult. These problems make it worthwhile to look at tools other than the traditionally used statistical approaches to develop a detection model. One of these potential tools is data mining, which is the process of finding new and potentially useful knowledge from existing large databases (Mitchell, 1999). Decision-tree induction (Quinlan, 1986) is a commonly used data mining technique that is often used for classification problems. Kamphuis et al. (2010a) reported a simple decision tree that was capable of detecting CM with a similar performance compared with detection models currently used by AMS. They suggested improving detection performance by using more CM cases and using quarter milkings (QM) that clearly had CM or were healthy in the training process. Also, combining decision-tree induction with bagging and boosting techniques was expected to improve detection performance (Kamphuis et al., 2010a).

The objective of this study was to develop and validate a CM detection model by means of decision-tree induction that meets the specific requirements when the model is applied in an AMS setting. One such specific requirement is that the model should detect at least severe cases of CM at a very high level of specificity. At the same time, it should be able to deal with QM with a less clear mastitis status, because most QM fall into this category in practice. Finally, a CM model should generate alerts for $\mathrm{CM}$ within a limited period around or preferably before a CM case.

\section{MATERIALS AND METHODS}

\section{Data Collection and Preparation}

Data were collected at 9 commercial dairy farms in the Netherlands using a total of 12 Lely Astronaut AMS [version A2 $(\mathrm{n}=10)$ or A3 $(\mathrm{n}=2)$; Lely Industries N.V., Maassluis, the Netherlands]. Farmers were selected for participation based on the primary criterion that they were dealing with $\mathrm{CM}$ detection seriously. As a prerequisite, farmers were expected to use the mastitis alert list daily and consistently to detect cows suspected of having CM. In addition, farmers had to have at least $1 \mathrm{yr}$ of experience using an AMS. Data collection began at 2 farms in November 2006. From October 2007 onward, data were collected on all 9 farms. Data collection continued until March 2009.

Data from the AMS were collected by installing remote computers on each AMS. These remote computers stored all data available during a cow milking. Data comprised the cow identification number, and date and time stamps of the cow's entrance, teat cup attachments, and start of milk flow. All raw sensor measurements were stored as well, after discarding the first $30 \mathrm{~mL}$ (approximately) of quarter milk. Raw sensor measurements included average electrical conductivity, red, green, and blue color values for each quarter milking and for each $100 \mathrm{~mL}$ of quarter milk produced, and an estimation of quarter milk yield. Quarter milkings where the AMS failed to connect the teat cup, those with missing data for teat cup attachment and 100$\mathrm{mL}$ measurements, and those without data at the QM level (e.g., sensor measurement averages, start milk flow, teat cup attachment) were deleted from the data set. For the remaining QM, recorded 100-mL values for electrical conductivity, and red, green, and blue that were outside the mean $\pm 4 \mathrm{SD}$ were regarded as likely data errors and were set as missing. Sensor data were combined with SCC data collected as part of the dairy herd improvement program. Because SCC was determined at the cow milking level, all QM within a cow milking were assigned the SCC value determined on a specific test-day for a specific cow.

Cows that raised suspicion of $\mathrm{CM}$ according to the criteria of the farmer were checked visually for hav- 
ing CM. The farmer's suspicion could be based on the mastitis alert lists, but also on other information such as SCC data or the presence of clots on the milk filter. This approach resulted in different procedures per farm to check quarters for CM. By introducing a scoring protocol, however, the assessment of the actual mastitis status of visually checked quarters was standardized. The scoring protocol instructed farmers to visually score the fifth and sixth squirts of milk of all quarters they checked using a clean black paddle as commonly used for the California Mastitis Test (without using the reagent normally used when applying this test). When visually normal, the milk was scored as 1 . When abnormal, the milk could be scored as follows: $2=$ watery milk, $3=$ flakes, $4=$ clots, $5=$ serum-like milk, or 6 $=$ milk with blood. Farmers were instructed to record the cow identification number, quarter, date and time, and the score assigned. Every 4 to $6 \mathrm{wk}$, farmers were visited to collect data from the remote computer and the scoring forms. During these visits, the scoring forms were discussed with the farmer to ensure proper use of the scoring protocol.

To combine visual observations of $\mathrm{CM}$ and sensor data, each visual quarter milk assessment was linked to sensor data from the most recent quarter milking, within a 24-h time window before the assessment time, recorded for that same quarter by the remote computer (situation A, Figure 1).

Data from the 4 sensors (electrical conductivity, and the red, green, and blue color sensors), 2 derived measurement patterns (average color value and milk flow), and 3 measurement patterns involving one single value
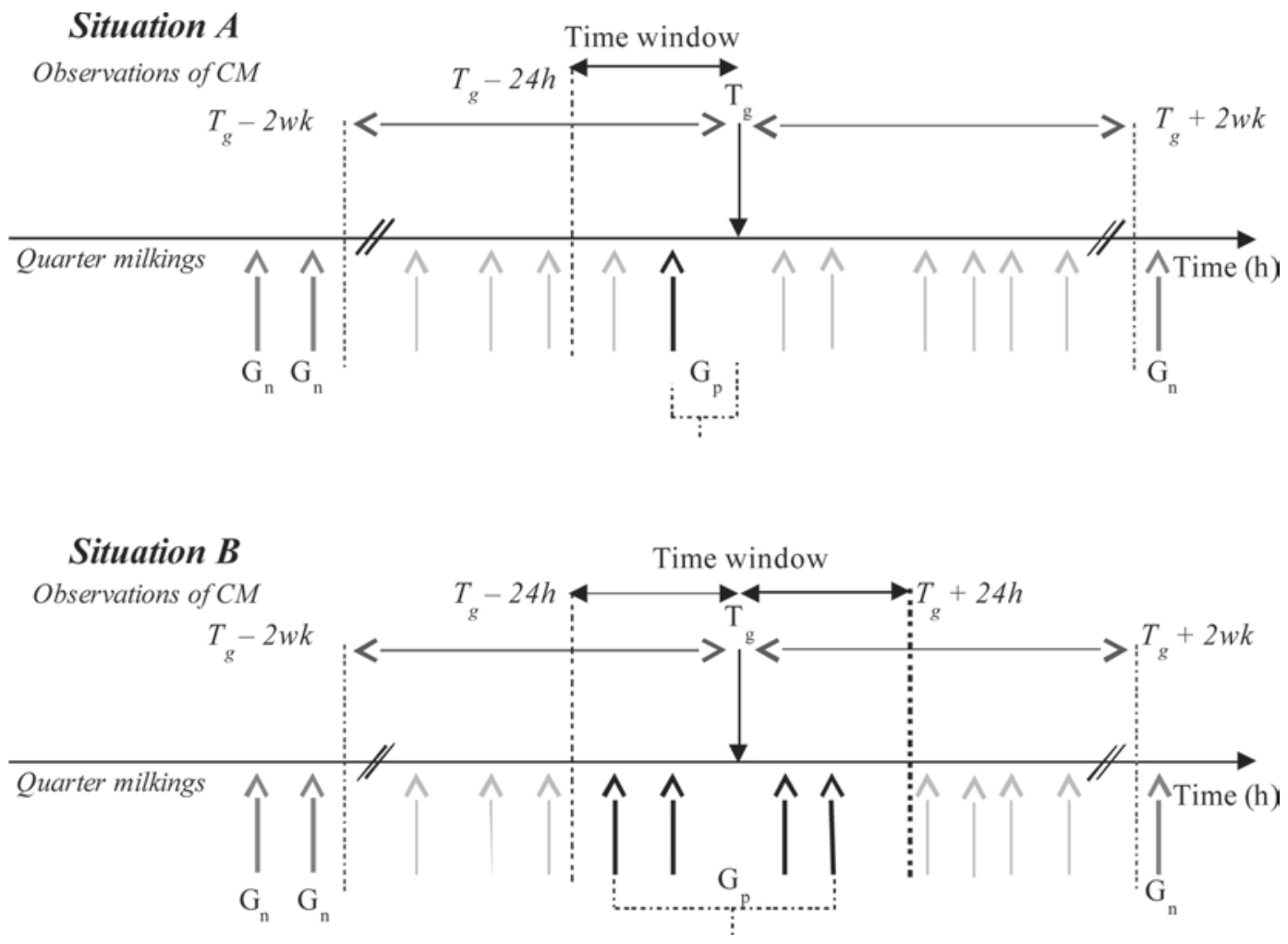

Figure 1. Combining an observation of clinical mastitis (CM) to a quarter milking recorded by the automatic milking system (situation A) and selecting quarter milkings to compute sensitivity when time windows are applied (situation B). Situation A: Combining an observation of a quarter with clinical mastitis $\left(\mathrm{T}_{\mathrm{g}}\right)$ with sensor data from the last milking before the observation recorded by the automatic milking system for that same quarter (black arrow pointing up). This first milking had to be within a 24-h time window before the observation and was considered gold standard positive $\left(G_{p}\right)$. This labeling of $G_{p}$ quarter milkings was similar for the training and the test set. For validation, quarter milkings within a 2-wk period around $T_{\mathrm{g}}$ were excluded from the data set (light gray arrows pointing up). Quarter milkings outside this 2-wk period (dark gray arrows pointing up) were considered gold standard negative $\left(\mathrm{G}_{\mathrm{n}}\right)$. Situation B: Example of implementing a time window of $24 \mathrm{~h}$ before and $24 \mathrm{~h}$ after the observation of clinical mastitis $\left(\mathrm{T}_{\mathrm{g}}\right)$ to label quarter milkings (black arrows pointing up) as gold standard positive $\left(\mathrm{G}_{\mathrm{p}}\right)$. Each of the $G_{p}$ quarter milkings receives a probability estimate for having clinical mastitis by the decision tree. Other quarter milkings inside a 2-wk period but outside the chosen time window are excluded from the data set (light gray arrows pointing up). Quarter milkings outside a 2-wk period around a $T_{g}$ (dark gray arrows pointing up) are considered gold standard negative $\left(G_{n}\right)$. The probabilities for clinical mastitis of the $G_{p}$ quarter milkings are compared with a threshold that results in a specificity of $99 \%$ or $97.9 \%$. If this probability for clinical mastitis exceeds this chosen threshold, that quarter milking receives an alert for clinical mastitis. If the number of alerts is $\geq 1$ in the specified time window, then this time window is counted as a true-positive alert in calculating sensitivity. If no alerts are assigned, then this time window is considered a false-negative alert in calculating sensitivity. 
for a whole QM (milk flow delay, dead milking time, and milk production) were used to define descriptive variables $(\mathrm{n}=1,065)$ as described in Kamphuis et al. (2008a, 2010a). These independent variables described characteristics (level, variability, and shape) of the sensor measurement patterns from each QM.

\section{Model Development}

Sensor data were available from 1,109 individual cows and almost 3.5 million QM. Participating farmers checked 1,593 QM visually for CM, of which 348 QM received a score from 2 through 5 . These were considered as gold standard positive QM and included $74 \mathrm{QM}$ with watery milk, $217 \mathrm{QM}$ with flakes, 50 QM with clots, and 7 serum-like QM. These 348 QM with CM out of almost 3.5 million QM give a rough estimate of $0.36 \mathrm{CM}$ cases per 365 cow-days at risk. There were also 6 QM containing blood (score 6). These were excluded from the data set because milk with blood does not necessarily imply CM but could be caused by damage in the udder or the teat. In addition, algorithms are already available to accurately detect milk with blood (Rasmussen and Bjerring, 2005). The data were divided into a training set (including two-thirds of all QM) and a test set. Cows in the training set could not be present in the test set and vice versa, and the number of $\mathrm{CM}$ cases in the training set was about two-thirds of all available QM with CM.

The training set included slightly more than 2.3 million QM from 738 cows. From these QM, 1,028 QM were combined with a visual observation. Only QM with SCC information from cows that never exceeded a SCC level of 200,000 cells/mL within the lactation and were never visually checked by the farmers during the study period were selected and labeled as gold standard negative. The final training set used for model development comprised 24,960 QM from 404 cows, of which 243 QM were labeled gold standard positive and 24,717 QM were labeled gold standard negative.

Before the CM detection model was developed, the 200 most informative independent variables were selected based on the information gain ratio (Quinlan, 1986; Witten and Frank, 2005; Kamphuis et al., 2008a). This method selects those variables that best discern between positive and negative cases of CM.

To develop a CM detection model, decision-tree induction was applied to the training set, consisting of the 200 selected independent variables and 24,960 QM. A decision tree is a graphic representation of a divideand-conquer approach of a classification problem and consists of nodes at which a variable is tested. The construction of a decision tree can be expressed recursively: based on its information gain ratio an indepen- dent variable is selected to split a data set at the first node. For each possible outcome of the test involved at that node, a branch is made ending in a daughter node. Next, the process can be repeated for each branch, using only those records that actually reach the branch. If at any time all records at a node have the same classification, that part of the tree stops developing (Witten and Frank, 2005). An example of a simplified and hypothetical decision tree is presented in Figure 2. A record in a test set will eventually flow into one of the classification nodes (colored gray in Figure 2), and the record will receive that classification output (CM or healthy). To build a decision tree, the J48 algorithm as implemented in WEKA (Witten and Frank, 2005) with default settings was used. This J48 algorithm was used on its own, but also in combination with bagging and boosting to study their effect on detection performance. Boosting and bagging are data mining techniques that can be used with any classification algorithm, such as the J48 algorithm, and both techniques operate by selectively resampling from the training set to generate derived training sets to which the basic algorithm is applied (Webb, 2000). In bagging, records are resampled in each iteration $t$, giving equal weight to each record in the training set. In forming the final classification, bagging also gives equal weight to each of the individual decision-tree models built at each iteration $t$. Boosting, on the other hand, uses reweighting of records in each iteration $t$, where records wrongly classified in the previous iteration $t-1$ receive more weight in the current iteration $t$. Finally, in forming the final classification, boosting gives a "good" model (one with fewer errors in the classification of records in the derived training sets) more weight than "bad" models (Freund and Schapire, 1996). In the current study, the number of iterations for bagging and boosting was set at 10. The Adaboost. M1 and the bagging algorithm in WEKA were used (Witten and Frank, 2005).

\section{Validation}

The decision tree developed was validated using data from the test set. This test set included over 1.1 million QM from 371 cows that did not appear in the training set. A total of 559 QM were visually checked by the farmers, of which 105 QM were labeled as gold standard positive. From this test set, QM at the cow milking level that lay within a 2-wk period around a QM with CM were deleted from the data set, including those QM without CM that appeared in the same cow milking that had a QM with CM. This selection resulted in the exclusion of $24,161 \mathrm{QM}$ (2.1\% of all QM in the test set). All other remaining QM were labeled as gold standard negative. This gold standard label- 
ing procedure is represented by situation A in Figure 1. From the large number of gold standard negative $\mathrm{QM}$, a random sample of 50,000 QM was selected. The 50,105 records in the final test set used for validation came from 368 individual cows.

The decision-tree model provided probability estimates for having CM for each QM in the test set. These probability estimates were compared with a threshold value to classify a QM as "healthy" or "CM." When the probability estimate exceeded the threshold, this QM was classified as CM. A gold standard positive QM that was classified by the decision-tree model as CM was considered a true-positive (TP) classification. When the decision tree classified a gold standard negative QM as healthy, this was considered a true-negative (TN) classification. A false-positive (FP) classification was a gold standard negative QM that was classified as CM, and finally, a false-negative $(\mathbf{F N})$ classification was a gold standard positive QM that was classified as healthy. These 4 classification types were used to compute the Se as the fraction of gold standard positive QM that were classified as CM $[\mathrm{Se}(\%)=100 \times \mathrm{TP} /$ $(\mathrm{TP}+\mathrm{FN})]$ by the decision tree, and the $\mathrm{Sp}$ as the fraction of gold standard negative $\mathrm{QM}$ that were classified as healthy $[\mathrm{Sp}(\%)=100 \times \mathrm{TN} /(\mathrm{TN}+\mathrm{FP})]$. To evaluate the detection performance of a decision-tree model, the Se was calculated at 2 fixed Sp levels: an Sp of $99 \%$ has been suggested by Mein and Rasmussen (2008) as a level that mastitis detection models should achieve when applied in practice. The second level of Sp was $97.9 \%$, which is an Sp level found by H. Mollenhorst and H. Hogeveen (2008, unpublished data), who studied the performance of CM detection models used currently by the AMS. As a result, the thresholds at which the probability estimates for having CM were compared were 0.442413 for an Sp of $99 \%$ and 0.282771 for an Sp of $97.9 \%$.

Receiver operating characteristic curves are graphic representations of the TP rate (or Se) versus the $\mathrm{FP}$ rate (or $1-\mathrm{Sp}$ ) over the whole range of possible threshold values (Detilleux et al., 1999). To summarize the receiver operating characteristic curve into a single quantity, the area under the curve (AUC) is a commonly used measure. However, for the classification problem at hand, total AUC is not an informative measure, because detection performance at high FP rates (resulting in too many $\mathrm{FP}$ alerts on the mastitis alert list) is of no interest. Therefore, the partial AUC (pAUC) was computed as a third measure to evaluate detection performance. The pAUC is restricted to just a relevant portion of the total AUC (Detilleux et al., 1999) and was calculated using the trapezoidal rule for Sp values of $\geq 99 \%$ and $\geq 97.9 \%$. To be able to use the same interpretation as the total AUC, the pAUC values

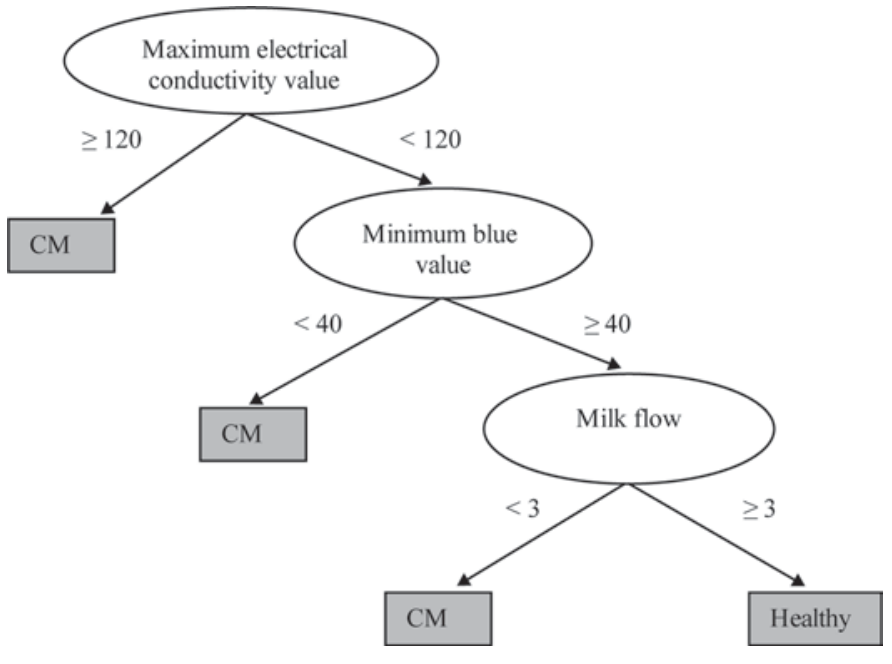

Figure 2. Example of a simplified and hypothetical decision tree using 3 independent variables based on sensor measurements to classify records into healthy or diseased (clinical mastitis, CM) quarter milkings.

were transformed to the same original scale that is used for the total AUC (McClish, 1989).

\section{Exploring Time Windows and Herd-Specific Performances}

As described above, detection performance was based on the labeling procedure depicted in situation $\mathrm{A}$ in Figure 1.This approach resulted in a very narrow time window $(<24 \mathrm{~h})$ in which an alert by the decision tree was considered TP or FN. The application of time windows has been described by Sherlock et al. (2008). Their suggestions were used as the basis for evaluating Se of the decision tree in the current study, when time windows were enlarged. The implementation of wider time windows is shown in situation B in Figure 1, where an example is presented of a time window of $24 \mathrm{~h}$ before to $24 \mathrm{~h}$ after an observation of CM. The main difference with situation A (Figure 1) is that not all QM within a 2-wk period were excluded and that not just one QM received a gold standard positive label. Instead, all QM of the affected quarter within the time window of $24 \mathrm{~h}$ before to $24 \mathrm{~h}$ after a CM observation were labeled gold standard positive, and all these QM received a probability estimate for having CM by the decision tree. Again, these probability estimates were compared with the same threshold values that resulted, for situation A (Figure 1), in an Sp of $99 \%$ or $97.9 \%$. If the probability estimate exceeded the chosen threshold, that specific QM received an alert for CM. If the number of alerts within the specified time window of $24 \mathrm{~h}$ before or after a $\mathrm{CM}$ observation was $\geq 1$, then this time window was considered a TP alert for computing Se. If none of the 
Table 1. Number of automatic milking systems (AMS) per herd, and number of unique cows, quarter milkings (QM), and clinical mastitis (CM) cases included in the training and test set per herd

\begin{tabular}{|c|c|c|c|c|c|c|c|}
\hline \multirow[b]{2}{*}{ Herd } & \multirow[b]{2}{*}{$\operatorname{AMS}(\mathrm{n})$} & \multicolumn{3}{|c|}{ Training set } & \multicolumn{3}{|c|}{ Test set } \\
\hline & & Cows (n) & $\mathrm{QM}(\mathrm{n})$ & $\mathrm{CM}(\mathrm{n})$ & Cows (n) & $\mathrm{QM}(\mathrm{n})$ & $\mathrm{CM}(\mathrm{n})$ \\
\hline 3 & $1^{1}$ & 28 & 1,568 & 3 & 27 & 2,840 & 2 \\
\hline 4 & $1^{1}$ & 41 & 1,221 & 5 & 38 & 2.779 & 2 \\
\hline 5 & 1 & 36 & 3,475 & 9 & 28 & 4,069 & 5 \\
\hline 8 & 2 & 62 & 6,294 & 19 & 67 & 10,430 & 9 \\
\hline 9 & 2 & 58 & 2,563 & 37 & 48 & 6,766 & 15 \\
\hline Total & 12 & 404 & 24,960 & 243 & 368 & 50,105 & 105 \\
\hline
\end{tabular}

${ }^{1}$ Herds with an A3 Lely Astronaut automatic milking system (Lely Industries N.V., Maassluis, the Netherlands). All other herds used an A2 Lely Astronaut automatic milking system.

selected QM received an alert, this time window was considered an FN alert in computing Se.

The decision tree was trained and tested using QM from all 9 herds; data between the training and test sets were thus correlated to some degree. Analyses were conducted to study the detection performance of a CM decision-tree model applied to truly unseen data; that is, to data from a herd that was not used for training at all. Therefore, decision-tree models were trained, using the same approach as described above, with data from 8 herds and validated with data from the ninth herd. This resulted in 9 different decision-tree models that were validated on 9 herds that were not used for training. When a decision tree was trained, all QM with $\mathrm{CM}$ of the 8 herds were used, and all QM with CM from the ninth herd were included in the test set.

Data preparation was done using SAS (version 9.1, SAS Institute Inc., Cary, NC). Development of several decision-tree models and probability estimates for having CM for each quarter milking were done in WEKA (version 3.4.8; Witten and Frank, 2005). Data produced in WEKA were used in SAS to retrieve the Se at the fixed Sp levels and to compute the transformed pAUC values. Decision-tree models were tested to determine whether Se levels, at the 2 fixed Sp levels, were statistically significantly different using the McNemar's test in SAS.

\section{RESULTS}

Table 1 shows the results of the stratification procedure to obtain QM used for training and testing. Because some cows had more than one CM case, the stratification at the cow level caused the CM cases not to be distributed strictly by two-thirds for training and one-third for testing. The number of $\mathrm{CM}$ cases varied between herds, with a maximum of $81 \mathrm{CM}$ cases observed in herd 2 and a minimum of $5 \mathrm{CM}$ cases in herd 3. The random sample of 50,000 gold standard negative QM were selected proportionally to the total number of gold standard negative QM per herd in the test set. The number of gold standard negative QM varied largely between herds. Herds 8 and 9 both had 2 AMS and data were collected over a similar period ( 2 full years), but herd 8 had substantially more gold standard negative QM than herd 9.

Table 2 shows the detection performance of 3 decisiontree models when validated on all 50,105 QM of the test set. All 3 models used the same 200 most informative independent variables, but differed in training procedure. The model that used the J48 algorithm alone showed an Se of $24.7 \%$ at an Sp of $99 \%$ and an Se of $43.8 \%$ at an $\mathrm{Sp}$ of $97.9 \%$. The pAUC was 0.556 for $\mathrm{Sp}$ levels of $\geq 99 \%$ and 0.612 for Sp levels of $\geq 97.9 \%$. When combining the J48 algorithm with boosting, there was an increase in both Se and pAUC at both Sp levels but this increase was only statistically significant at an Sp of $99 \%(P=0.003)$, and not at an Sp of $97.9 \%(P=$ 0.532 ). Values for Se and pAUC were highest (Se of $40.0 \%$ and $57.1 \%$ for the 2 fixed Sp levels of $99 \%$ and $97.9 \%$, respectively, and pAUC of 0.639 and 0.693 for Sp levels of $\geq 99 \%$ and $\geq 97.9 \%$, respectively) for the decision-tree model that combined the J48 algorithm with bagging. The Se of this decision-tree model was statistically significantly different from that of the decision tree that used the J48 alone at both Sp levels $(P$ $=0.0002$ at an Sp of $99 \%$, and $P=0.004$ at an Sp of $97.9 \%)$. The difference in Se between the decision trees that used either bagging or boosting in combination with the J48 algorithm was statistically significant $(P$ $<0.0001)$ at an $\mathrm{Sp}$ of $97.9 \%$. Therefore, the decision tree that combined the J48 algorithm with bagging was chosen as the best model and is referred to as the base decision tree hereafter. All other results are based on 
Table 2. Sensitivity (Se) at a specificity of $99 \%\left(\mathrm{Se}^{99}\right)$ and a specificity of $97.9 \%\left(\mathrm{Se}^{97.9}\right)$, and transformed partial area under the curve for specificity levels $\geq 99 \%\left(\mathrm{pAUC}^{99}\right)$ and $\geq 97.9 \%\left(\mathrm{pAUC}^{97.9}\right)$ of 3 decision-tree models $^{1}$

\begin{tabular}{lcccc}
\hline Decision-tree model & $\mathrm{Se}^{99}$ & $\mathrm{pAUC}^{99}$ & $\mathrm{Se}^{97.9}$ & $\mathrm{pAUC}^{97.9}$ \\
\hline Using all test data (50,105 quarter milkings) & & & & \\
J48 & $24.7^{\mathrm{a}}$ & 0.556 & $43.8^{\mathrm{a}}$ & 0.612 \\
J48 with boosting & $38.1^{\mathrm{b}}$ & 0.636 & $52.4^{\mathrm{ab}}$ & 0.681 \\
J48 with bagging & $40.0^{\mathrm{bc}}$ & 0.639 & $57.1^{\mathrm{c}}$ & 0.693 \\
Using J48 with bagging on herd-specific test data & & & & \\
By herd & & & & \\
1 & 38.5 & 0.624 & 76.9 & 0.70 \\
2 & 23.8 & 0.577 & 23.8 & 0.596 \\
3 & 0.0 & 0.498 & 0.0 & 0.495 \\
4 & 50.0 & 0.613 & 50.0 & 0.682 \\
5 & 60.0 & 0.759 & 60.0 & 0.779 \\
6 & 71.4 & 0.846 & 71.4 & 0.851 \\
7 & 18.2 & 0.560 & 36.4 & 0.592 \\
8 & 66.7 & 0.783 & 77.8 & 0.822 \\
9 & 13.3 & 0.564 & 46.7 & 0.602 \\
\hline
\end{tabular}

${ }^{\text {a-c }}$ Values within the same column with different superscripts are statistically significantly different $(P<$ $0.05)$.

${ }^{1}$ One model used the J48 algorithm alone, one combined J48 with boosting, and one combined J48 with bagging. Detection performance values are computed using all test data $(50,105$ quarter milkings; 105 with clinical mastitis) and per herd.

this base decision tree. When applying the base decision tree to herd-specific data in the test set, it became clear that detection performance varied largely between herds. Herds 1, 6, and 8 showed a high Se at an Sp of $97.9 \%$ (also reflected by a high pAUC for $\mathrm{Sp} \geq 97.9 \%$ ). At a higher Sp (99\%), the base decision tree still performed well on herds 6 and 8 . The base decision tree did not perform well on herds 2, 7, and 9. Performance of the base decision tree was not informative for herds 3,4 and 5 because of the very low number of $\mathrm{CM}$ cases in the test set for these herds (see also Table 1).

The test set included 105 QM with CM, of which 16 were scored as watery milk, 72 contained flakes, 14 contained clots, and 3 were serum-like. Table 3 summarizes which $\mathrm{CM}$ cases were detected by the base decision tree at the 2 fixed Sp levels. The base decision tree detected 42 QM with CM at an Sp of $99 \%$ and 60 QM with CM at an Sp of $97.9 \%$, coinciding with the Se levels of $40.0 \%$ and $57.1 \%$ reported earlier (Table 2). The base decision tree had problems detecting watery milk, with only $12.5 \%$ of these cases detected at an Sp of $99 \%$ and $37.5 \%$ at an Sp of $97.9 \%$. Most CM cases were observed as milk containing flakes $(68.6 \%)$, and at an $\mathrm{Sp}$ of $97.9 \%$ the base decision tree alerted for more than half of these cases. There were 14 more severe CM cases, indicated by clots in the milk. At an Sp of $99 \%$, the model detected $64.3 \%$ of these cases. This percentage increased to $78.6 \%$ at an $\mathrm{Sp}$ of $97.9 \%$.

Table 4 shows the Se at the 2 fixed Sp levels of the base decision tree when different time windows were

Table 3. The number of quarter milkings with clinical mastitis (CM) detected by the base decision-tree model at 2 fixed levels of specificity (99\% and 97.9\%), stratified by the score the observed CM received by the participating farmers

\begin{tabular}{lccccc}
\hline & \multicolumn{4}{c}{ CM observations receiving score (n) } \\
\cline { 2 - 5 } Specificity level (\%) & $\begin{array}{c}\text { Score 2 } \\
\text { (watery milk) }\end{array}$ & $\begin{array}{c}\text { Score 3 } \\
\text { (flakes) }\end{array}$ & $\begin{array}{c}\text { Score 4 } \\
\text { (clots) }\end{array}$ & $\begin{array}{c}\text { Score 5 } \\
\text { (serum-like milk) }\end{array}$ & Total (n) \\
\hline 99 & 2 & 30 & 9 & 1 & 42 \\
& $(4.8)^{1}$ & $(71.4)$ & $(21.4)$ & $(2.38)$ & $(40.0)$ \\
97.9 & $(12.5)^{1}$ & $(41.7)$ & $(64.3)$ & $(33.3)$ & 60 \\
& 6 & 41 & 11 & 2 & $(3.3)$ \\
Total & $(10.0)$ & $(68.3)$ & $(18.3)$ & $(66.7)$ & 105 \\
& $(37.5)$ & $(56.9)$ & $(78.6)$ & $14.1)$ \\
\hline
\end{tabular}

${ }^{1}$ First value in parentheses indicates the percentage from the row total; the second value in parentheses indicates the percentage from the column total. 
applied. The first row in the table shows the Se of the base decision tree when applied to the time window that was used to combine CM observations with sensor data from the AMS. A considerable increase in Se was found when a time window of $24 \mathrm{~h}$ before or after a CM observation was applied: Se increased to $66.7 \%$ at an Sp of $99 \%$ and to $79 \%$ at an $\mathrm{Sp}$ of $97.9 \%$. Results showed that Se increased as the selected time windows were widened, and that not all CM were detected by the base decision tree, even when very wide time windows were applied: slightly more than $10 \%$ of the $\mathrm{CM}$ cases were not detected when a time window of $10 \mathrm{~d}$ (or 240 h) before to $7 \mathrm{~d}$ (or $168 \mathrm{~h}$ ) after the CM observation was applied at an Sp of $97.9 \%$.

Results in Table 5 report the Se and pAUC of 9 decision trees built on data from 8 herds and tested on data from the ninth herd. As in Table 2, the Se varied largely between herds and, for some herds, there was a large difference in Se at the 2 fixed levels of Sp. Again, detection performance was not very informative for herds 3 and 4 because of the small numbers of QM with CM used for testing. The Se deteriorated for some herds (e.g., herds 6 and 8) at both Sp levels compared with the Se levels reported in Table 2, which were based on a decision tree built with data from all 9 herds. For example, the Se of herd 6 decreases by $18 \%$ at an Sp of $99 \%$, and a decrease in Se of $13.5 \%$ is seen for herd 8 at an Sp of $97.9 \%$. In addition, there was a considerable decrease in pAUC, with a maximum decrease in pAUC of 0.150 . On the other hand, herd 9 showed an increase in Se of $21.3 \%$ at an Sp of $99 \%$ when the decision tree was trained on data from 8 other herds. The increase was, however, less pronounced in the pAUC that increased by 0.043 . In this specific herd, Se did not increase at an Sp of $97.9 \%$.

\section{DISCUSSION}

In recent years, several studies on automated detection of CM have been published. Most of these studies collected detailed information on a relatively small number of CM cases, and data were mostly collected from one (research) herd. In the current study, a CM detection model was developed using sensor and observational data collected from 9 commercially operating Dutch dairy herds milking automatically, which is a unique approach in the field of CM research. The approach was chosen deliberately to collect data on a large number of CM cases $(n=348)$ in a relatively short period, without intervening with the daily working routine of the farmers involved. A rough estimate of the $\mathrm{CM}$ incidence of the current study $(0.36 \mathrm{CM}$ cases per 365 cow-days at risk) was similar to the $\mathrm{CM}$ incidence rate of 0.31 per 365 cow-days at risk found in a survey of Dutch dairy farmers (Van den Borne et al., 2008). This confirmed the idea that the CM observations by the participating farmers in the current study were reliable and representative for the true CM status in their herds.

The base decision tree differed statistically $(P<$ $0.05)$ from the decision-tree model that applied the J48 algorithm alone at both Sp levels (97.9 and 99\%) and from the decision-tree model that combined the J48 algorithm with boosting at an Sp of $97.9 \%$. When compared with previously conducted studies on CM detection models that report both an Se and an Sp, the base decision tree showed improved detection performance. For example, a recent study conducted by Kramer et al. (2009) reported on a CM detection model with an Sp of $92 \%$ and an Se of $75 \%$ using a time window of $4 \mathrm{~d}$ before the CM observation and the day of observation.

Table 4. Sensitivities (Se) of the base decision tree at a specificity of $99 \%\left(\mathrm{Se}^{99}\right)$ and at a specificity of $97.9 \%$ $\left(\mathrm{Se}^{97.9}\right)$ using different time windows

\begin{tabular}{lcccc}
\hline \multicolumn{2}{c}{ Used time window $(\mathrm{h})$} & & \multicolumn{2}{c}{ Detection performance } \\
\cline { 1 - 2 } Before CM observation & After CM observation & & $\mathrm{Se}^{99}$ & $\mathrm{Se}^{97.9}$ \\
\hline$<24^{1}$ & 24 & & 40.0 & 57.1 \\
24 & 24 & & 66.7 & 79.0 \\
$48^{2}$ & 48 & & 73.5 & 81.0 \\
$96^{3}$ & 72 & & 75.2 & 82.9 \\
96 & 168 & & 70.5 & 85.7 \\
$120^{4}$ & 0 & & 84.8 & 89.5 \\
$240^{5}$ & & & & \\
\hline
\end{tabular}

${ }^{1}$ Based on the single quarter milking that was combined with the observation score of clinical mastitis $(\mathrm{CM})$. This single quarter milking was the first quarter milking before the observation of CM, but had to be within a 24 -h period before the observation.

${ }^{2}$ Time window applied in Sherlock et al. (2008).

${ }^{3}$ Time window applied in Claycomb et al. (2009).

${ }^{4}$ Time window applied in Kramer et al. (2009).

${ }^{5}$ Time window applied in de Mol et al. (1997). 
Table 5. Total number of quarter milkings (QM) and the number of QM with clinical mastitis (CM) used for training decision-tree models, total number of QM and the number of QM with CM used for validating the decision-tree models, the sensitivity at a specificity of $99 \%\left(\mathrm{Se}^{99}\right.$ ) and a specificity of $97.9 \%\left(\mathrm{Se}^{97.9}\right)$, and the transformed partial area under the curve for specificity levels $\geq 99 \%\left(\mathrm{pAUC}^{99}\right)$ and $\geq 97.9 \%\left(\mathrm{pAUC}^{97.9}\right)^{1}$

\begin{tabular}{|c|c|c|c|c|c|c|}
\hline $\begin{array}{l}\text { Decision-tree model } \\
\text { validated using } \\
\text { data from herd }\end{array}$ & $\begin{array}{l}\text { Number of QM } \\
\text { (with CM) } \\
\text { for training }\end{array}$ & $\begin{array}{l}\text { Number of QM } \\
\text { (with CM) } \\
\text { for validation }\end{array}$ & $\mathrm{Se}^{99}$ & $\mathrm{pAUC}^{99}$ & $\mathrm{Se}^{97.9}$ & $\mathrm{pAUC}^{97.9}$ \\
\hline 1 & $21,780(271)$ & $7,332(77)$ & 41.6 & 0.654 & 51.9 & 0.692 \\
\hline 3 & $23,495(343)$ & $2,843(5)$ & 0.0 & 0.498 & 0.0 & 0.495 \\
\hline 4 & $23,842(341)$ & $2,784(7)$ & 57.1 & 0.599 & 57.1 & 0.694 \\
\hline 5 & $21,585(334)$ & $4,078(14)$ & 64.3 & 0.744 & 64.3 & 0.783 \\
\hline 6 & $22,886(290)$ & $3,869(58)$ & 53.4 & 0.696 & 62.0 & 0.741 \\
\hline 9 & $22,487(296)$ & $6,803(52)$ & 34.6 & 0.609 & 44.2 & 0.650 \\
\hline
\end{tabular}

${ }^{1}$ All decision trees are trained with data from 8 herds using the J48 algorithm combined with bagging (200 best independent variables were selected using information gain ratio) and validated with data from the ninth herd.

The base decision tree developed in the current study reached an Se of $92.4 \%$ at the same Sp when this time window of 5 d was applied. Norberg et al. (2004) applied a stricter time window, where only the milking at which a CM was observed was of interest for the CM detection model to alert for, and reported an Se of $43 \%$ at an Sp of $93 \%$ to detect CM. The time window was similar to the one used here $(<24 \mathrm{~h})$, and fixing the Sp at $93 \%$, the base decision tree in the current study showed an Se of $75.2 \%$. The base decision tree showed an Se of $57.1 \%$ at an Sp of $97.9 \%$. This is an increase in Se of more than 20 percentage points compared with detection models currently used by AMS at the same Sp of 97.9\% (H. Mollenhorst and H. Hogeveen; unpublished data, 2008). Farmers are eager to minimize false-positive alerts even at the expense of a lower Se (Claycomb et al., 2009). When interpreting results from the farmer's perspective, the base decision tree was able to keep the Se at the same level as currently used models ( $40 \%$ for the base decision tree vs. $36.8 \%$ ) while decreasing the number of false-positive alerts by more than $50 \%$ as Sp increased from 97.9 to $99 \%$.

The application of time windows, in which an alert by a detection model is considered a true-positive alert, was previously implemented by de Mol et al. (1997). The discussion about the effect of implementing time windows of different lengths on detection performance is more recent. Sherlock et al. (2008) presented some guidelines for performance evaluation when implementing different time windows. They reported improved detection performance when time windows were wider. This trend was seen in the current study as well: the Se from the base decision tree increased by more than 20 percentage points at both Sp levels when the time window increased from $<24 \mathrm{~h}$ before a $\mathrm{CM}$ observation to a 24-h time window before or after a $\mathrm{CM}$ observation (Table 4). Although detection models that use large time windows for their alerts will show higher levels of Se and Sp than models using smaller time windows, there is a risk that these detection models may lose their practical use (e.g., the time window of $10 \mathrm{~d}$ before to $7 \mathrm{~d}$ after a CM reported by de Mol et al. (1997). What time window is useful for a CM detection model applied in an AMS setting remains debatable: use a wider time window for early detection, including the risk that visual signs of $\mathrm{CM}$ are not there at the time of an alert or use a time window for detection of quarters with clear visible signs of $\mathrm{CM}$ with the risk of alerts for cows already being clinically infected for a few days? Keefe et al. (2010) summarized results from studies using on-farm culture results to apply selective antibiotic treatment. They report no differences in clinical or bacteriological cure rates between cows treated with antibiotics directly after a CM occurrence and cows that received a selective antibiotic treatment based on on-farm culture tests with results typically available within $24 \mathrm{~h}$ after the CM occurrence. This may suggest that alerts by a CM detection model after the CM occurrence itself do not necessarily result in worse cure rates. It seems sensible, however, to assume that time windows should be as narrow as possible, although it is not implied that the narrow time window of $<24 \mathrm{~h}$ used in the current study is the correct one.

Validation on a data set that includes only QM that are clearly healthy or CM as done by Friggens et al. (2007) will give an overestimated performance. They reported high levels of Se and Sp, but their detection model would probably not reach this level of performance in practice because in reality not all QM are clearly healthy or CM. The effect of including or excluding QM with a less clear CM status has been studied by Kamphuis et al. (2010b). They reported a decrease in Se of 25 percentage points when validation was applied to a test set that included QM with a less clear mastitis 
status, compared with a test set that used only QM that were clearly healthy or CM. The base decision tree from the current study was trained using QM that were clearly healthy or CM. In doing so, the amount of noise in the training set was decreased, which in return was expected to ease the training process for the decision-tree algorithm. On the other hand, validation was applied to data that mimicked practice as closely as possible, because a large number of QM with a less clear mastitis status were kept in the validation set. By selecting a random sample of 50,000 QM, it was expected that all potential variation of sensor measurements of these QM with a less clear mastitis status was captured and that validation on such a test set would better reflect detection performance of models when they are implemented in practice.

Another point of debate is the necessary Se and Sp levels a CM detection model should have when it is implemented in practice. For visual observation of CM or abnormal milk, several levels for Se have been suggested. Hillerton (2000) reported an Se of $80 \%$ for visual inspection of milk for CM. Rasmussen (2005) suggested an Se of $70 \%$ as a reasonable level to detect abnormal milk during foremilking using a filter with pore size of $0.1 \mathrm{~mm}$. The International Organization for Standardization (ISO, 2007) suggests an Se of $70 \%$ for automatic milking installations. This Se level is reported as the lower confidence interval value using an $80 \%$ CI. When applying this lower confidence interval value in combination with an Sp level of $99 \%$, suggested by Mein and Rasmussen (2008) as minimum level for practical use, the base decision tree should reach an Se of $75 \%$ at an Sp of $99 \%$. Using the short time window used in the current study $(<24 \mathrm{~h})$, however, the base decision tree showed an Se of $40 \%$ at an Sp of $99 \%$. To meet the requirements mentioned, it was necessary to widen the time interval to $4 \mathrm{~d}$ before to $3 \mathrm{~d}$ after a CM observation (Table 4). The question remains whether this wide time window is still useful for a practical AMS setting.

Results showed that the base decision tree performed well on some herds (e.g., herds 1, 6, and 8) but poorly on others (e.g., herds 2 and 7; Table 2). Based on our experience when collecting data during the farm visits, the idea arose that farmers from these "bad" herds were more chaotic in their daily working routines than farmers from "good" herds. They did detect CM cases, but it seemed that they detected these cases by using information sources other than the mastitis alert lists and that they did not use the information presented on the mastitis alert list in a systematic and consistent way to detect CM. On the other hand, farmers from "good" herds seemed to be well organized and seemed to have developed a strict protocol of using the information presented on the mastitis alert list, without using a lot of additional information. Including data from "bad" herds in the training set may introduce unwanted noise, which makes it more difficult for the decision-tree algorithm to develop a good detection model. Similarly, including data from "good" herds in the training set may be very useful in developing a generic CM detection model. These ideas were reflected by results reported in Table 5: "bad" herds showed a low detection performance for models that were trained with uncorrelated data, suggesting that these farmers indeed detected the $\mathrm{CM}$ cases by means other than the mastitis alert lists. "Good" herds showed lower Se levels of the models trained on uncorrelated data compared with models based on partly correlated data, potentially indicating a loss of useful information when data from these herds were excluded for training. This observation raised the question of whether it would be better to train herd specific models for these "good" herds. For example, when building a herd-specific model for herd 1 (using only data from that herd), the herd-specific decision tree showed an Se of $76.9 \%$ at an Sp of $97.9 \%$ (data not shown). This is a similar Se compared with that of the base decision tree model, but the herd-specific decision tree was trained with only $55 \mathrm{QM}$ with $\mathrm{CM}$, about onefourth of the QM with $\mathrm{CM}$ on which the base decision tree was based. It is expected that performance would increase if the herd-specific model were trained using more CM cases, which suggests that a herd-specific model would be very useful for these "good" herds.

Claycomb et al. (2009) indicated that it should be possible for farmers to vary thresholds of future detection models to adapt them to their own specific preferences for detecting TP cases or minimizing FP alerts. This is an interesting idea, and it would be a useful addition for farmers compared with current detection models where the options to customize are minimal. It should be realized, however, that this would require the automatic milking industry to explain to farmers, as end-users of the detection model, the effects of changing these thresholds and the interrelationship between Se and Sp. Suppose the farmer of herd 1 (Table 5) changes the threshold from the decision tree so that $\mathrm{Sp}$ decreases from 99 to $97.9 \%$. At the same time, Se would increase from 41.6 to $51.9 \%$. In absolute numbers, the model would now detect 8 additional CM cases at the expense of 80 extra FP alerts. The farmer might be satisfied with this change in FP and TP alerts. For herd 8 , however, this change in threshold values would increase the number of FP alerts by more than 100 , but just 1 additional CM case would be detected. This would probably be a much less attractive change in TP and FP alerts. A customizable CM detection model is 
an attractive idea, but it should be applied carefully by farmers and might not be an attractive additional option for all farmers.

The base decision tree detected reasonably well $\mathrm{CM}$ cases that were observed as having flakes or clots (Table 3 ) when using the narrow time window of $<24 \mathrm{~h}$ : more than $56 \%$ of CM cases with flakes and almost $80 \%$ of CM cases with clots were detected at an Sp of $97.9 \%$. At an Sp of 99\%, 64\% of all CM cases with clots were detected. Unfortunately, the base decision tree was not able to detect all severe cases of CM (score 4), and the detection of watery milk was very poor, with only $12.5 \%$ detection at an $\mathrm{Sp}$ of $99 \%$. This indicates that using sensor data (electrical conductivity, color, and milk flow) alone is not enough to detect all CM cases, and other information sources are necessary to guarantee that all cases are found by farmers.

To evaluate detection performance, the Se was computed at 2 fixed Sp levels (99 and 97.9\%). These evaluators are an estimate of the true detection performance of the developed decision trees because not all QM and not all alerts on the mastitis alert list were checked visually. Because of this lack of continuous monitoring of all QM for CM, alerts are generated by the decisiontree model that may have been true-positive for CM but are now considered false-positive or in alerts that may have been false-negative but are considered now truenegative. Consequently, the number of true-positive and false-negative alerts may have been underestimated and the number of false-positive and true-negative alerts may have been overestimated. However, the choice to exclude QM within a 2-wk period around a CM observation (resulting in an exclusion of $2.1 \%$ of all $\mathrm{QM}$ in the test set) was made to prevent mislabeling of QM that already had CM but that were not detected earlier by the farmers or mislabeling of QM with subclinical mastitis as gold standard negative. In addition, the selection procedure for farmers required them to use the mastitis alert lists daily and consistently. By taking these measures, the number of missed true-positive alerts was kept as low as possible. This problem was also encountered by Steeneveld et al. (2010). They conducted a study in which cow information was combined with alerts from the mastitis alert list to judge whether these alerts were true-positives or false-positives. Just as in the current study, they used the selection criterion that herd managers worked seriously in detecting CM cases. In addition, they noted that it is likely that missed cases of CM cured spontaneously or that they were detected later when infection had deteriorated (Steeneveld et al., 2010), two points that are applicable to the current study as well.

\section{CONCLUSIONS}

Decision-tree induction made it possible to maintain Se at about the same level as models currently used by AMS but to decrease the number of FP alerts by more than $50 \%$. It was also possible to increase Se from 36.8 to $57.1 \%$ when the Sp level was kept at the level of models currently used by AMS (97.9\%). The base decision tree is expected to be suitable for use in practice for the following reasons: data from 9 commercially producing dairy herds were used for model development and validation, alerts for CM were given within a very short time window $(<24 \mathrm{~h})$, and finally, validation was conducted on a test set that included QM with a less clear mastitis status, mimicking practice as closely as possible.

\section{ACKNOWLEDGMENTS}

We gratefully acknowledge the participating farmers for their contribution to the acquisition of the data used in this research. Also gratefully acknowledged is Lely Industries N.V., Maassluis, the Netherlands) for providing addresses of farmers working with AMS and their support with the installation of the data acquisition systems at the participating farms. This research is supported by the Dutch Technology Foundation STW, Applied Science division of NOW, and the Technology Program of the Ministry of Economic Affairs.

\section{REFERENCES}

Claycomb, R. W., P. T. Johnstone, G. A. Mein, and R. A. Sherlock. 2009. An automated in-line clinical mastitis detection system using measurement of conductivity from foremilk of individual udder quarters. N. Z. Vet. J. 57:208-214.

de Koning, K., B. Slaghuis, and Y. van der Vorst. 2004. Milk quality on farms with an automatic milking system. Pages 311-320 in Automatic Milking-A Better Understanding. A. Meijering, H. Hogeveen, and C. J. A. M de Koning, ed. Wageningen Academic Publishers, Wageningen, the Netherlands.

de Mol, R. M., G. H. Kroeze, J. M. F. H. Achten, K. Maatje, and W. Rossing. 1997. Results of a multivariate approach to automated oestrus and mastitis detection. Livest. Prod. Sci. 48:219-227.

Detilleux, J., J. Arendt, F. Lomba, and P. Leroy. 1999. Methods for estimating areas under receiver-operating characteristic curves: Illustration with somatic-cell scores in subclinical intramammary infections. Prev. Vet. Med. 41:75-88.

Dohmen, W., F. Neijenhuis, and H. Hogeveen. 2010. Relationship between udder health and hygiene on farms with an automatic milking system. J. Dairy Sci. 10.3168/jds.2010-3028.

Freund, Y., and R. E. Schapire. 1996. Experiments with a new boosting algorithm. Pages 148-156 in Proceedings of the Thirteenth International Conference of Machine Learning. L. Saitta, ed. Morgan Kaufmann Publishers, San Francisco, CA.

Friggens, N. C., M. G. G. Chagunda, M. Bjerring, C. Ridder, S. Hojsgaard, and T. Larsen. 2007. Estimating degree of mastitis from time-series measurements in milk: A test of a model based on lactate dehydrogenase measurements. J. Dairy Sci. 90:54155427 . 
Halasa, T., K. Huijps, O. Osteras, and H. Hogeveen. 2007. Economic effects of bovine mastitis and mastitis management: A review. Vet. Q. 29:18-31.

Hillerton, J. E. 2000. Detecting mastitis cow-side. Pages 48-53 in National Mastitis Council 39th Annual Meeting. Atlanta, GA. Natl. Mastitis Counc., Madison, WI.

Hogeveen, H., and W. Ouweltjes. 2003. Sensors and management support in high-technology milking. J. Anim. Sci. 81(Suppl. $3): 1-10$.

ISO. 2007. Automatic milking systems - requirements and testing. Annex C: Example of methods of evaluating detection systems for milk deemed as abnormal due to blood or to changes in homogeneity. ISO 20966:2007. International Organization for Standardization, Geneva, Switzerland.

Kamphuis, C., H. Mollenhorst, A. J. Feelders, D. Pietersma, and H. Hogeveen. 2010a. Decision-tree induction to detect clinical mastitis with automatic milking. Comput. Electron. Agric. 70:60-68.

Kamphuis, C., H. Mollenhorst, J. A. P. Heesterbeek, and H. Hogeveen. 2010b. Data mining to detect clinical mastitis with automatic milking. Pages 568-572 in Proceedings of the 5th IDF Mastitis Conf. J. E. Hillerton, ed. The New Zealand Veterinary Association Foundation for Continuing Education Inc., Wellington, New Zealand.

Kamphuis, C., D. Pietersma, R. van der Tol, M. Wiedemann, and H. Hogeveen. 2008a. Using sensor data patterns from an automatic milking system to develop predictive variables for classifying clinical mastitis and abnormal milk. Comput. Electron. Agric. $62: 169-181$

Kamphuis, C., R. Sherlock, J. Jago, G. Mein, and H. Hogeveen. 2008b. Automatic detection of clinical mastitis is improved by in-line monitoring of somatic cell count. J. Dairy Sci. 91:4560-4570.

Keefe, G., J. McCarron, K. MacDonald, and M. Cameron. 2010. The scientific bases for using on-farm culture systems. Pages 141-148 in Proceedings of the 49th Annual Meeting of the National Mastitis Council. Albuquerque, NM. Natl. Mastitis Counc., Madison, WI.

Kramer, E., D. Cavero, E. Stamer, and J. Krieter. 2009. Mastitis and lameness detection in dairy cows by application of fuzzy logic. Livest. Sci. 125:92-96.
McClish, D. K. 1989. Analyzing a portion of the ROC curve. Med. Decis. Making 9:190-195.

Mein, G. A., and M. D. Rasmussen. 2008. Performance evaluation of systems for automated monitoring of udder health: Would the real gold standard please stand up? Pages 259-266 in Mastitis Control-From Science to Practice. T. J. G. M. Lam, ed. Wageningen Academic Publishers, Wageningen, the Netherlands.

Mitchell, T. M. 1999. Machine learning and data mining. Commun. ACM 42:30-36.

Norberg, E., H. Hogeveen, I. R. Korsgaard, N. C. Friggens, K. H. M. N. Sloth, and P. Lovendahl. 2004. Electrical conductivity of milk: Ability to predict mastitis status. J. Dairy Sci. 87:1099-1107.

Quinlan, J. R. 1986. Induction of decision trees. Mach. Learn. 1:81106.

Rasmussen, M. D. 2005. Visual scoring of clots in foremilk. J. Dairy Res. 72:406-414.

Rasmussen, M. D., and M. Bjerring. 2005. Visual scoring of milk mixed with blood. J. Dairy Res. 72:257-263.

Sherlock, R., H. Hogeveen, G. Mein, and M. D. Rasmussen. 2008. Performance evaluation of systems for automated monitoring of udder health: Analytical issues and guidelines. Pages 275-282 in Mastitis Control-From Science to Practice. T. J. G. M. Lam, ed. Wageningen Academic Publishers, Wageningen, the Netherlands.

Steeneveld, W., L. C. d. Gaag, W. Ouweltjes, H. Mollenhorst, and H. Hogeveen. 2010. Discriminating between true-positive and falsepositive clinical mastitis alerts from automatic milking systems. J. Dairy Sci. 93:2559-2568. doi:10.3168/jds.2009-3020.

Van den Borne, B. H. P., G. Van Schaik, T. J. G. M. Lam, and M. Nielen. 2008. A comparison of the occurrence of mastitis in Dutch primi- and multiparous cows. Pages 55-62 in Mastitis ControlFrom Science to Practice. T. J. G. M. Lam, ed. Wageningen Academic Publishers, Wageningen, the Netherlands.

Webb, G. I. 2000. Multiboosting: A technique for combining boosting and bagging. Mach. Learn. 40:159-196.

Witten, I. H., and E. Frank. 2005. Data Mining; Practical Machine Learning Tools and Techniques. 2nd ed. I. H. Witten and E. Frank, ed. Morgan Kaufmann Publishers, San Francisco, CA. 